\title{
Analysis of Importance of Components in Power Systems using Time Sequential Simulation
}

\author{
G. Vancells ${ }^{1}$, S. Herraiz ${ }^{1}$, J. Meléndez ${ }^{1}$ and Á. Ferreira ${ }^{2}$ \\ ${ }^{1}$ eXiT, Control Engineering and Intelligent Systems Research Group. \\ Institute of Informatics and Applications, University of Girona \\ Campus of Montilivi - Av. Lluís Santaló, 17071 Girona (Spain) \\ Phone number: +34 972418761, e-mail: \{gerard.vancells; sergio.herraiz; joaquim.melendez\}@udg.edu \\ ${ }^{2}$ Indra Sistemas \\ Av. de Bruselas, 3528108 Alcobendas. Madrid (Spain) \\ Phone number: +34 914809157, e-mail: aferreirar@indra.es
}

\begin{abstract}
Power systems reliability analysis consists in evaluating the ability of a system to perform its function without failures. Usually, it is measured in failures per year $(\lambda)$. In the present work, the reliability of the global system is used to obtain the importance of components in order to identify which parts of the network must be improved or which components are the less reliable and need to be upgraded. The objective is to increase the global reliability of the system by introducing better components or redundant branches to guarantee the best quality of the power in the load points. A Time Sequential Monte Carlo simulation has been implemented in Matlab® and tested in a HV/MV substation to determine the importance of its components.
\end{abstract}

\section{Key words}

Reliability, Components Importance Indexes, Power systems, Monte Carlo simulation.

\section{Introduction}

According to the IEEE, the reliability of a system is defined as the ability of the system to perform its function under stated conditions for a period of time without failures [1]. The basic reliability indexes are the failure rate $(\lambda)$, the average outage time $(r)$ and time average annual outage time $(U)$.

The reliability of power systems has received special attention in recent years, especially the reliability of distribution systems, mainly because it directly affects consumers, but the first studies are from the 30s. A detailed description of the reliability theory in power systems can be found in [2] [3] [4].

The evaluation methods are divided in two groups: analytical methods based on mathematical models and simulation approaches based on Monte Carlo simulation, either sequentially or non-sequentially. Analytical methods have been historically the most widely used and include the network modeling and Markov methods [5]. Simulation methods are taking on greater importance in recent times due to the improvement of calculation ability of computers, essential for analyze large complex networks. A comparison between both methods is described in [5].

In this paper, the reliability of an electrical substation is evaluated, considering that its main function is to meet the demand as economical as possible, ensuring a reasonable level of quality and continuity in service.

A time sequential simulation based on Monte Carlo simulation has been implemented in order to determine the importance of components of a power substation. Monte Carlo Time sequential simulations are based in MTTF (Mean Time to Failures) and MTTR (Mean Time to Restoration). Therefore a set of historical data of MTTF and MTTR values is needed. In the literature some works give typical values that can be used [6].

Monte Carlo simulation method has been implemented in Matlab® software, and the components of a distribution substation evaluated to determine the most important ones in terms of reliability. As we have seen in the literature, there are some types of indexes that we can use. Indexes give us different information about some aspects of the component and the system. Some of them are interesting in the design stage to identify critical parts, while some others are useful for doing the maintenance of the net in an advanced stage of operation.

The paper is organized as follows: Time sequential simulation is explained in Section II. Importance Indexes are presented in Section III. Analysis of a substation is performed in Section IV. Conclusions and future work are presented in Section V.

\section{Time sequential simulation}

Before the calculation of the Monte Carlo simulation a set of historical reliability data for each component is needed. This reliability values set can be formed with 
static (non-time-varying) or dynamic (time-varying) reliability values. In the static way the reliability values of components are from 0 to 1 , and it is necessary to generate a random value and compare it with the historically reliability value of each component (Fig.1).

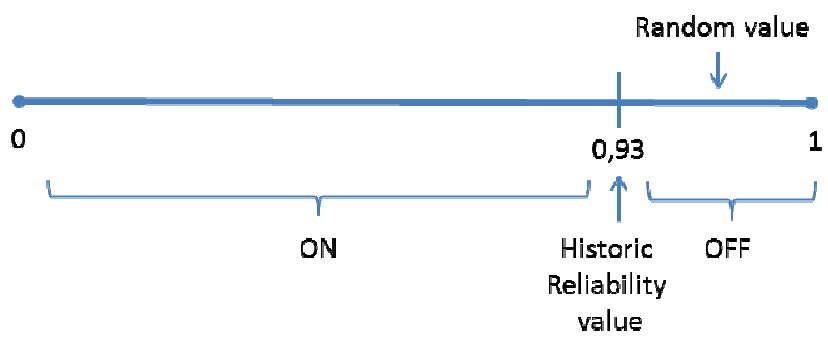

Fig. 1. Determination of the state of a component

The dynamic way uses MTTF and MTTR, exponentially or normally distributed, depending whether we consider a constant failure rate or components with aging and an increasing failure rate during time.

These values are used to build a sequence of functioning history for each component, as shown in Fig. 2.

\section{COMPONENT}

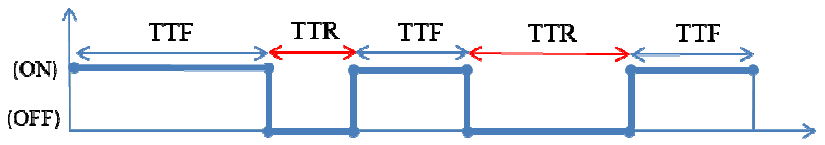

Time of simulation

Fig. 2. History of a component

Usually, the reliability values of components are very high. In terms of MTTF, it could be expressed in decades or centuries. Typical values of MTTR and MTTF for different components can be found in [6] and some of them are shown in Table 1.

Table 1. MTTF and MTTR typical values

\begin{tabular}{ccc}
\hline Components & MTTF (years) & MTTR (hours) \\
\hline Transformers & $40-70$ & 1300 \\
Switches & 48 & 60 \\
Disconnector & 38 & 10 \\
Cables & 29 & 40 \\
\hline
\end{tabular}

The MTTF and MTTR values are used to determine de TTF (Time to Failure) and TTR (Time to Restoration) values of each component. Considering a time constant failure rate the exponential distribution (1) can be used.

$$
f(x)= \begin{cases}\lambda e^{-\lambda x} & x \geq 0 \\ 0 & x<0\end{cases}
$$

Where $\lambda$ is a scale factor

Weibull distribution allows us to use non-time constant failure rates, for example, when having components aging or infant mortality.

The Monte Carlo simulation needs to calculate the stochastic values of TTF and TTR for all components at the first simulation step. These values are obtained using an exponential distribution. Also, the TTF and TTR values must be recalculated every time that a component is repaired, to determine the next failure instant.

Some studies indicate that exponential distribution is adequate for TTF and log-normal distributions could be more reasonable for TTR.

\section{Importance Indexes}

There are different indexes for assessing the importance of system components. The most used are based in the customer unreliability, while some others are focused in the cost of the service interruptions. Here, five typical importance indexes that deal with the global system reliability and unreliability are described.

\section{A. Birnbaum Index $\left(I^{B}\right)$}

The Birnbaum index of components importance (1969) consists in performing the partial derivate of the system reliability respect to the component $i$ failure rate, so that this ratio depends only on the structure of the system and the reliability of the other components, being independent from the own reliability of component $i$.

Otherwise, this index can be defined as the probability that the system is in a state such that the component $i$ is critical. As a weakness of this index, it should be noted that the index does not depend on the inner reliability of the component. This means that the component $i$ may be part of a minimum cut set of level 1, or a higher level where all the other components have already failed.

$$
I^{B}(i \mid t)=\frac{\partial h(\boldsymbol{p}(t))}{\partial p_{i}(t)}
$$

Where:

$i:$ is the component to evaluate.

$h(\boldsymbol{p}(t)):$ is the system reliability.

$p_{i}(t):$ is the reliability of component $i$.

This index is specially used in analytical methods and it is important because other indexes such as Critical Importance Index are based on it.

\section{B. Improvement Potential Index $\left(I^{P}\right)$}

The Improvement Potential Index of a component evaluates the system reliability increase if the component is replaced by a perfect one (with reliability $=1$ ).

The difference between both system reliability values (before and after the replacement) is called system reliability improvement potential.

Actually it is not possible to improve the reliability of the component to 1 . Therefore, it may be useful to evaluate the reliability of the system using a component with the maximum reliability that we can be found in the market. 


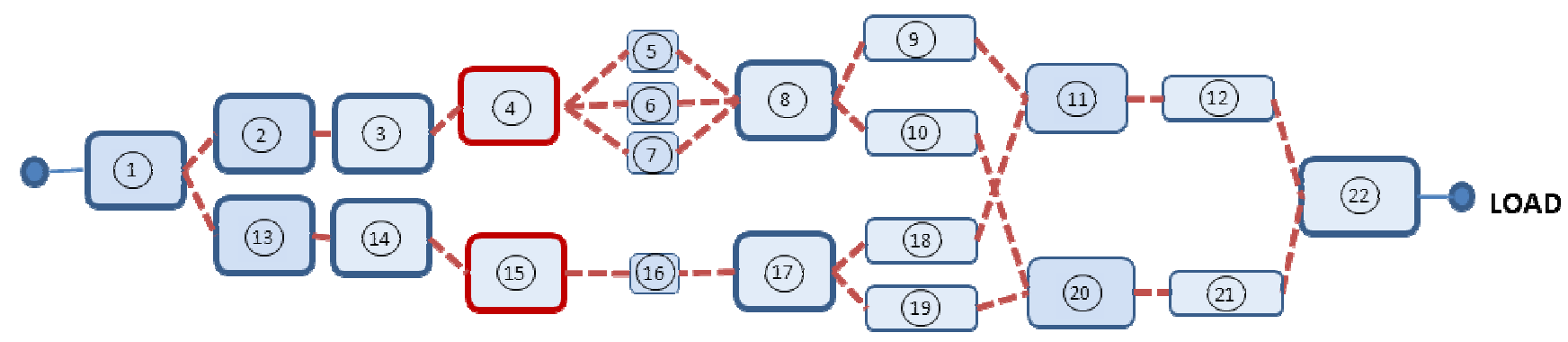

Fig. 3. Block diagram of the HV/MV substation

When the component reliability is 1 ,

$$
I^{I P}(i \mid t)=h\left(1_{i}, \boldsymbol{p}(t)\right)-h(p(t))
$$

When the component reliability is the best possible,

$$
I^{I P}(i \mid t)=h\left(\text { best }_{i}, \boldsymbol{p}(t)\right)-h(p(t))
$$

Where:

$h\left(1_{i}, \boldsymbol{p}(t)\right)$ : is the system reliability with the component reliability $\mathrm{i}=1$.

$h(p(t))$ : is the initial system reliability.

\section{Risk Reduction Worth Index (IRRW)}

This index is the ratio of the current system unreliability with the unreliability of the system considering a component $i$ with perfect reliability (equal to 1), at the instant of time $t$.

$$
I^{R R W}(i \mid t)=\frac{1-h(p(t))}{1-h\left(1_{i}, p_{i}(t)\right)}
$$

Where:

$h\left(1_{i}, \boldsymbol{p}(t)\right)$ : is the system reliability with the component reliability $\mathrm{i}=1$.

$h(p(t)):$ is the initial system reliability.

\section{Risk Achievement Worth Index $\left(I^{R A W}\right)$}

This index is the ratio of the conditional system unreliability if component $i$ is always failed with the failure rate of current system. It indicates the importance of maintaining the current level of component reliability for system reliability.

Where:

$$
I^{R A W}(i \mid t)=\frac{1-h\left(0_{i}, \boldsymbol{p}(t)\right)}{1-h\left(p_{i}(t)\right)}
$$

$h\left(0_{i}, \boldsymbol{p}(t)\right)$ : is the system reliability with the component reliability $\mathrm{i}=0$.

$h(\boldsymbol{p}(t)):$ is the initial system reliability.

\section{E. Critical Importance Index (ICI)}

This index is related with Birnbaum Index. It is a measure of the importance of components that is particularly useful for maintenance prioritization actions.
The component $i$ is critical for the system if the other system components are in such a state that the system is working if and only if component $i$ is working. $I^{C R}(i / t)$ also is the probability that a component $i$ cause a system failure.

$$
I^{C R}(i \mid t)=\frac{I^{B}(i \mid t) \cdot\left(1-p_{i}(t)\right)}{1-h(p(t))}
$$

Where:

$1-h(\boldsymbol{p}(t)):$ System failure rate.

$1-h\left(p_{i}(t)\right):$ Component failures $\boldsymbol{i}$.

\section{Analysis of a substation}

Importance of the components of a HV/MV substation has been evaluated using time varying Monte Carlo simulation. This method allows getting the importance indices of the components from different points of view. The importance of the components has to be assessed to ensure supply at the load point with a reasonable level of quality and continuity.

\section{A. Power substation}

Fig.3 shows the single line diagram of the power substation used in this work as an example to implement the reliability method. Table 2 shows the types of components.

Table 2. Type of HV/MV substation components

\begin{tabular}{cccl}
\hline Comp. & type & Comp. & \multicolumn{1}{c}{ type } \\
\hline $\mathbf{1}$ & Bar & $\mathbf{1 2}$ & Disconnector \\
$\mathbf{2}$ & Disconnector & $\mathbf{1 3}$ & Disconnector \\
$\mathbf{3}$ & Switches & $\mathbf{1 4}$ & Switches \\
$\mathbf{4}$ & Transformer & $\mathbf{1 5}$ & Transformer \\
$\mathbf{5}$ & Cable & $\mathbf{1 6}$ & Cable \\
$\mathbf{6}$ & Cable & $\mathbf{1 7}$ & Switches \\
$\mathbf{7}$ & Cable & $\mathbf{1 8}$ & Disconnector \\
$\mathbf{8}$ & Switches & $\mathbf{1 9}$ & Disconnector \\
$\mathbf{9}$ & Disconnector & $\mathbf{2 0}$ & Bar \\
$\mathbf{1 0}$ & Disconnector & $\mathbf{2 1}$ & Disconnector \\
$\mathbf{1 1}$ & Bar & $\mathbf{2 2}$ & Switches \\
\hline
\end{tabular}

The HV/MV substation is double-bar type and is divided into two voltage zones, $220 \mathrm{kV}$ and $45 \mathrm{kV}$.

A history circuit status must be constructed using the MTTF and MTTR values of the components. In our case, the used MTTF and MTTR values have been adapted 
from those shown in Table 1 to allow a considerable number of failures and be able to determine the importance of components using a feasible number of simulations. The reliability values used in this work are shown in Table 3.

Table3. MTTF and MTTR values used

\begin{tabular}{ccc}
\hline Components & MTTF (hours) & MTTR (hours) \\
\hline Bars & - & - \\
Transformers & 650 & 10.16 \\
Switches & 160 & 10.7 \\
Disconnector & 7500 & 10 \\
Cables & 150 & 10.7 \\
\hline
\end{tabular}

\section{B. Time-Varying Monte Carlo simulation}

A set of 500 simulations of 1000 hours each of them were performed with Monte Carlo simulation method. As a result, a global reliability of $88.9 \% \pm 0.20 \%$ in the load point was obtained. This result is more optimistic than others because the simulation MTTF and MTTR includes the values and not only the value of the overall reliability of the component.

The following indexes are calculated and compared in this paper.

- Critical Importance Index $\left(\mathrm{I}^{\mathrm{CI}}\right)$

- Improvement Potential $\left(\mathrm{I}^{\mathrm{P}}\right)$

- Risk Reduction Worth Index $\left(\mathrm{I}^{\mathrm{RRW}}\right)$

- Risk Achievement Worth Index $\left(\mathrm{I}^{\mathrm{RAW}}\right)$

\section{B.1 Critical Importance Index $\left(I^{C I}\right)$}

Usually some components cause the major quantity of failures in a system. Critical Importance Index $\left(\mathrm{I}^{\mathrm{CI}}\right)$ determines the most harmful components to the system. Fig.4 show how only six components cause more than $95 \%$ of the failures of the system. With the improvement of the reliability of these components the reliability of the system will be improved as well.

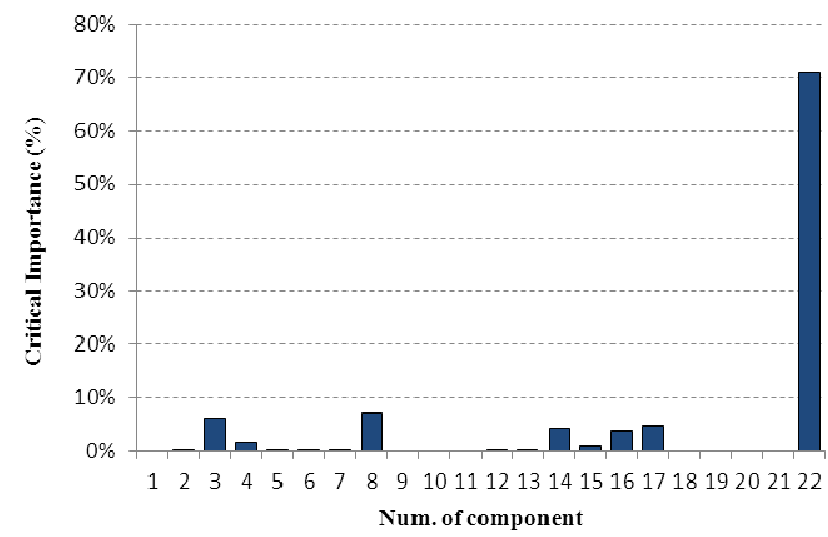

Fig. 4. Time-varying rank of components importance

The results of Fig. 4 show how the most important component from the point of view of reliability is component number 22. The reason is because this component is in series with the rest of the components, so every failure of this component will cause a system failure and a supply interruption to the load point.

Component number 1 is also in series with the rest of the system but in this case the component is a bar that it is considered a perfect component (reliability $=1$ ). Therefore it is like consider that this component can not fail.

\section{B.2 Improvement Potential $\left(I^{P}\right)$}

This index evaluates the enhancement of the system reliability if the component is replaced by one with reliability equal to 1 . The system reliability improvement is shown in Fig. 5 in terms of absolute percentage.

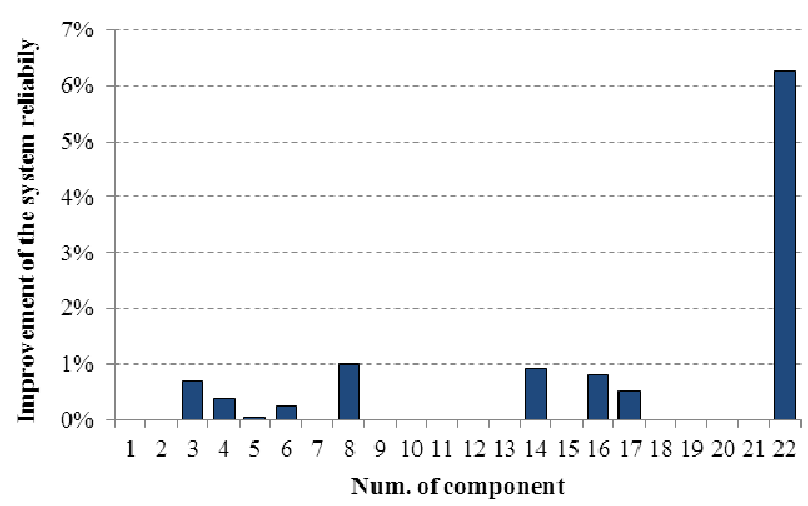

Fig. 5. Improvement of the system reliability

The component that can best improve the overall system reliability is number 22 , because this component is in series with the rest of the system.

The improvement of system reliability if this component cannot fail is around 6\%, and it increases the reliability from $88.9 \%$ to $94.9 \%$. The number 1 is also in series but in this case we had supposed that it could not fail, so this component already had reliability equal to 1 . Some other important components for this system are 8, 14, 16, 3, 17, 4 and 6.

\section{B.3 Risk Reduction Worth Index $\left(I^{R R W}\right)$}

The improvement of the system reliability also can be evaluated using ratios or the value of the system unreliability, considered after and before the replacement of the components for a perfect one (reliability=1). In Fig. 6 the values of $I^{R R W}$ index is plotted.

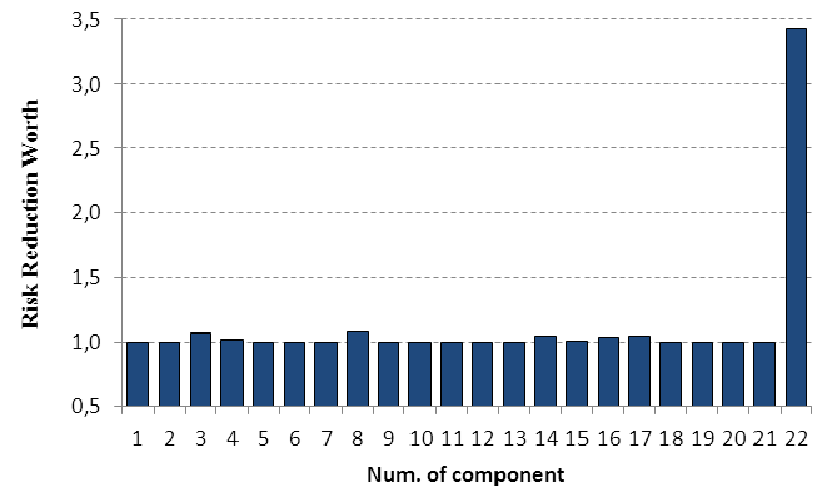

Fig. 6. Ratio of increment of system reliability 
In Fig. 6 component 22 continues being the most important, followed by $3,8,14,16$ and 17 very close of these last. In this case

\section{B.4 Risk Achievement Worth Index $\left(I^{R A W}\right)$}

The increase of system unreliability is evaluated with this index. To perform this evaluation we have to consider each component permanently failed. That means with reliability equal to 0 . Then the unreliability of the rest of the system is obtained and plotted as a ratio in Fig. 7 .

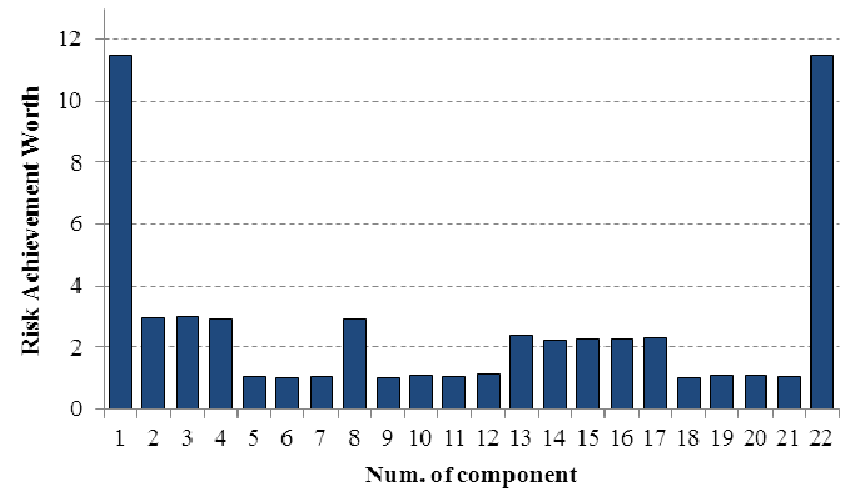

Fig. 7. Ratio of increment of system unreliability

Fig. 7 shows the importance of the components due only to the topological situation, instead of its own historical reliability mixed with topological situation like Critical Importance Index $\left(\mathrm{I}^{\mathrm{CI}}\right)$ and others. This index is interesting in a design stage because is possible think in duplicate some components, at least the ones with worst situation.

Also the system reliability in the load point can be obtained supposing each component failed and showed as an absolute percentage value instead as a ratio (Fig. 8).

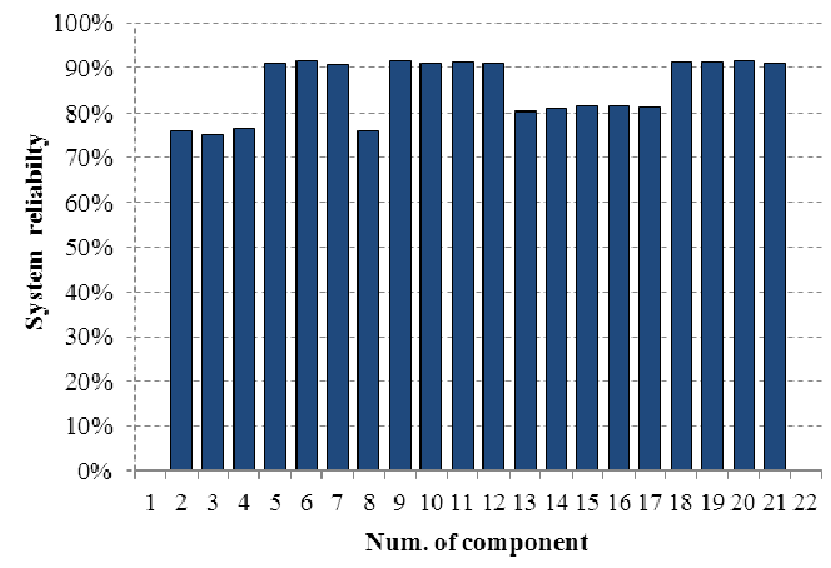

Fig. 8. System reliability with components in failure

The most important components from this point of view are sorted $(1,22,2,3,4,8,13,14,15,16$ and 17). The system reliability could be less of $80 \%$ if one of these components was failed. Therefore, it might be interesting try to prevent system failures due to one of these components by modifying the system in the design stage and trying to make them less critical when system works.

\section{Conclusions}

In this paper, a time sequential simulation was performed to assess the importance of the components in a power system. The existing importance indexes indicate the importance of each component according to different interests. A component can be important because its failure causes a complete failure of the system, which is called a critical failure. With this index it is possible to calculate the number of failures that every component causes. Other indexes are important because an improvement of the component reliability causes a better reliability of the system or can cause a decrease of the global reliability when it is non-operating.

The results show how the principal factor that affects the component importance in a power system is a combination of the topological situation and the initial and historical reliability of the component. In this sense, for example, two components with similar locations and different reliability values will have different importance indexes.

Finally, although time sequential simulations have a computational cost higher than non-time sequential simulations, they provide more optimistic and realistic results about the reliability of the system because it uses a most accurate way to calculate the functioning and nonfunctioning times for each component.

\section{Acknowledgment}

This work was supported by the research project "ENERGOS: Tecnologías para la gestión automatizada e inteligente de las redes de distribución energética del futuro" (http://innovationenergy.org/energos/) led by Gas Natural Fenosa (VDTI-CENIT with reference CEN20091048) funded by Ministerio de Economía y Competitividad (Spain) and "Monitorización inteligente de la calidad de la energía eléctrica" (DPI2009-07891, Ministerio de Ciencia e Innovación, Spain).

\section{References}

[1] C. A. Warren, "Distribution reliability: what is it?", $39^{\text {th }}$ Annual Conference in Rural Electric Power, 1995.

[2] R. Billinton and R.N. Allan, "Power-system reliability in perspective," Electronics \& Power, 1984.

[3] R. Billinton and R.N. Allan, "Probabilistic assessment of power systems", Proceedings of the IEEE, Vol. 88, No. 2, 2000, pp. 140-162.

[4] M. V. Pereira and N.J. Balu, "Composite generation/ transmission reliability evaluation", Proceedings of the IEEE, Vol. 80, No. 4, 1992, pp. 470-491.

[5] O. Shavuka, K. Awodele and S. Chowdhury, "Reliability Analysis of Distribution Networks", International Conference on Power System Technology (POWERCON), 2010.

[6] M. Bollen, Literature Search for Reliability Data of Components in Electric Distribution Networks, Eindhoven, TEUEDE, 1993. 